\title{
Waterlike Features, Liquid-Crystal Phase and Self-Assembly in Janus Dumbbells
}

\author{
José Rafael Bordin ${ }^{1}$ \\ 1-Campus Caçapava do Sul, Universidade Federal do Pampa, Av. Pedro Anunciação, 111, CEP 96570-000, Caçapava do Sul, RS, \\ Brazil \\ E-mail:josebordin@unipampa.edu.br
}

\begin{abstract}
We use Molecular Dynamics simulations to explore the properties of dimeric Janus nanoparticles. The nanoparticles are modeled as dumbbells. One kind of monomer interacts by a Lennard Jones potential, while the other specie of monomer interacts though a two length scale potential. This specific two length scale potential do not present waterlike anomalies in bulk. However, our results shows that the combination of teo length scales potential and LJ potential in the Janus nanoparticle will lead to thermodyncamic and dynamic anomalies. The self-assembly properties were also explored. We observe distinct kinds of self-assembled structures and a liquid-crystal phase. This results indicates that is possible to create Janus nanoparticles with waterlike features using monomers without anomalous behavior. The anomalies and structures are explained with the two length scale potential characteristics.

Keywords: Waterlike anomalies, Anomalous fluids, Janus dumbbells, self-assembly
\end{abstract}

\section{Introduction}

Most part of the materials contracts on cooling at constant pressure. Also, we expect that the diffusion coefficient will decrease when the pressure, or density, of the system is increased. However, this is not the case from the so called anomalous fluids. The most well known anomalous fluid is water [1, 2], with 73 known anomalies [3]. Liquid water at $1.0 \mathrm{~atm}$ have a maximum in the density at temperature of $4^{\circ} \mathrm{C}$, expanding as is cooled to $0^{\circ} \mathrm{C}$. Others materials, as silicon [4], silica [5], Te [6], Bi [7], Si [8], Ge 15 Te 85 [9], liquid metals [10], graphite [11] and $\mathrm{BeF}_{2}$ [12] also presents thermodynamic anomalies. Water [13], silicon [14] and silica [4] show a anomalous diffusion, characterized by a maximum in the diffusion coefficient at constant temperature.

Core-softened (CS) potentials with two length scales (TLS) have been widely used to study the general properties and characteristics of fluids with anomalous behavior [15, 16, 17, 18]. TLS potentials are characterized by having two preferred particle-particle separations, while one length scale (OLS) potentials, as the Lennard Jones (LJ) potential, shows only one preferred separation.

TLS potentials are able to reproduce waterlike anomalies in qualitative way if exists competition between the two characteristic distances $[17,19,20]$. However, if the energy penalty to the particle move 
from one scale to another is higher than the particle kinetic energy, the particle will get trapped in one length scale, and there will be no competition and, as consequence, there will be no anomalous behavior.

Another system of interest are colloidal suspensions. Despite the absence of anomalous behavior, TLS potentials were obtained from experiments using inversion of structural data of colloidal systems [21, 22]. Since such systems do not show waterlike anomalies, they can be studied using a TLS potential without competition between the scales.

Particularly, colloidal Janus nanoparticles have attract the attention of scientists due the large range of applications of this new materials, as applications in medicine, catalysis, photonic crystals, stable emulsions, biomolecules and self-healing materials [23, 24, 25, 26, 27, 28]. Dumbbells colloids are formed by two spheres that overlap with a separation that varies from an almost total overlap to one or two monomer diameters. The molecule anisotropy plays quite a relevant role. The properties of the system depend on the interaction potential that varies with their spatial separation and their relative orientations. In the case of Janus dumbbells [29, 30, 31, 32], each monomer have distinct characteristics, as charged/neutral or hydrophilic/hydrophobic. The competition between attractive and repulsive forces lead to the formation of self-assembly lamellae or micellae phases [33].

Recently, the production of silver-silicon ( $\mathrm{Ag}-\mathrm{Si})[30]$, silica-polystyrene $\left(\mathrm{SiO}_{2}-\mathrm{PS}\right)[34]$ and tantalum silicide-silicon $\left(\mathrm{TaSi}_{2} / \mathrm{Si}\right)[35]$ hybrid Janus dimers were reported. Silicon and silica are classified as anomalous fluids, and the silicon-silicon or silica-silica interaction in the pure system can be modeled by a TLS potential with competition between the scales. The others monomers can be described by an one length scale potential and consequently does not show the presence of the waterlike thermodynamic and dynamic anomalies. In our previous work we have show that, if the TLS potential shows dynamical and thermodynamical anomalous behavior for the monomeric case, the Janus dimer will have anomalies accordingly with the non-anomalous monomer properties [36].

A new question arises when none of the monomers are anomalous, but one can be modeled with a TLS potential without competition between the scales. For instance, soft colloidal [37, 38], metallic/polymer [39, 40] and liquid-crystal/polymer [41] Janus dumbbells have a colloidal monomer whose interaction can be described by a TLS potential [42] and a monomer that have OLS interaction.

In order to answer this question we explore the pressure versus temperature phase diagram of a model systems. The system is a composed by Janus particles in which one monomer interacts through a TLS potential without anomalies and the other monomer interacts through a LJ potential. We investigate how the presence of the LJ monomer affects the competition between the two characteristic distances, the self- 
assembly structures and describe qualitatively the thermodynamic phases.

The paper is organized as follows. The model, the methods and simulation details are described in the Section 2, the results and discussion are given in the Section 3, and then the conclusions are presented in the Section 4

\section{The Model and the Simulation details}

In this paper all the physical quantities are computed in the standard LJ units [43],

$$
r^{*} \equiv \frac{r}{\sigma}, \quad \rho^{*} \equiv \rho \sigma^{3}, \quad \text { and } \quad t^{*} \equiv t\left(\frac{\varepsilon}{m \sigma^{2}}\right)^{1 / 2},
$$

for distance, density of particles and time, respectively, and

$$
p^{*} \equiv \frac{p \sigma^{3}}{\varepsilon} \quad \text { and } \quad T^{*} \equiv \frac{k_{B} T}{\varepsilon}
$$

for the pressure and temperature, respectively, where $\sigma$ is the distance parameter, $\varepsilon$ the energy parameter and $m$ the mass parameter. Since in the present work all physical quantities are expressed in reduced LJ units we will omit the $*$ for simplicity.

$N$ dimers were used in each simulation, in a total of $2 N$ particles. Each monomer have diameter $\sigma$ and mass $m$, and the Janus dumbbells are constructed using two monomers linked rigidly at a distance $\lambda=\sigma$. The only difference between the monomers are their interaction. Monomers of type A interacts with another A monomer through a TLS core-softened potential, [17]

$$
\frac{U^{\mathrm{TLS}}\left(r_{i j}\right)}{\varepsilon}=4\left[\left(\frac{\sigma}{r_{i j}}\right)^{12}-\left(\frac{\sigma}{r_{i j}}\right)^{6}\right]+u_{0} \exp \left[-\frac{1}{c_{0}^{2}}\left(\frac{r_{i j}-r_{0}}{\sigma}\right)^{2}\right]-u_{1} \exp \left[-\frac{1}{c_{1}^{2}}\left(\frac{r_{i j}-r_{1}}{\sigma}\right)^{2}\right],
$$

where $r_{i j}=\left|\vec{r}_{i}-\vec{r}_{j}\right|$ is the distance between two A particles $i$ and $j$. This equation has three terms: the first one is the standard LJ 12-6 potential [43], the second is a Gaussian centered at $r_{0}=0.70$, with depth $u_{0}=5.0$ and width $c_{0}=1.0$, response for the shoulder in the potential shape, and the last term is also a Gaussian, but centered at $r_{1}=3.0$, with depth $u_{1}=-1.0$ and width $c_{1}=0.5$, responsible for the attractive tail of the potential, as indicated in figure 1 by the solid red line. Despite the presence of the two length scales, monomeric systems interacting through this potential did not shows waterlike anomalies since there was not observed competition between the scales [17]. B monomers interacts though a LJ potential,

$$
U^{\mathrm{LJ}}\left(r_{i j}\right)=\frac{4}{3} \cdot 2^{2 / 3}\left[\left(\frac{\sigma}{r_{i j}}\right)^{24}-\left(\frac{\sigma}{r_{i j}}\right)^{6}\right],
$$


the blue dashed line in figure 1. This particular shape for the LJ potential was chosen due to his small attractive well. Interactions between A and B monomers are also given by equation 4

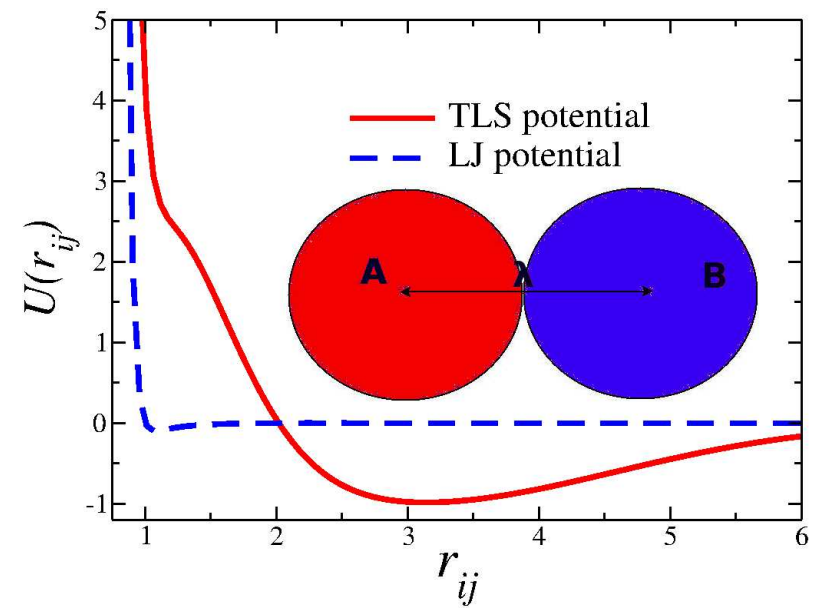

Figure 1: Interaction potentials used in our simulations: the TLS potential (solid red line) and the LJ potential (dashed blue line). Inset: Janus nanoparticles formed by A-B monomers.

NVT Molecular dynamics simulations using the ESPResSo package [44, 45] were performed in order to obtain the pressure versus temperature $(p \times T)$ phase diagram. A total of $N=1200$ particles were used in our simulations. To ensure our results, simulations with up to 9000 dimers where carried out, and essentially the same results were obtained. The number density is defined as $\rho=N / V$, where $V=L^{3}$ is the volume of the cubic simulation box. Standard periodic boundary conditions are applied in all directions. The system temperature was fixed using the Langevin thermostat with $\gamma=1.0$. The equations of motion for the fluid particles were integrated using the velocity Verlet algorithm, with a time step $\delta t=0.01$. The cutoff radius for the TLS potential is $r_{\text {cutTLS }}=6.5$, and $r_{\text {cutLJ }}=2.5$ for the LJ potential. We performed $5 \times 10^{6}$ steps to equilibrate the system. These steps are then followed by $5 \times 10^{6}$ steps for the results production stage. To ensure that the system was equilibrated, the pressure, kinetic energy and potential energy were analyzed as function of time, as well several snapshots at distinct simulation times.

To study the dynamic anomaly the relation between the mean square displacement (MSD) with time is analyzed, namely

$$
\left\langle\left[\vec{r}_{\mathrm{cm}}(t)-\vec{r}_{\mathrm{cm}}\left(t_{0}\right)\right]^{2}\right\rangle=\left\langle\Delta \vec{r}_{\mathrm{cm}}(t)^{2}\right\rangle,
$$

where $\vec{r}_{\mathrm{cm}}\left(t_{0}\right)=\left(x_{\mathrm{cm}}\left(t_{0}\right)^{2}+y_{\mathrm{cm}}\left(t_{0}\right)^{2}+z_{\mathrm{cm}}\left(t_{0}\right)^{2}\right)^{1 / 2}$ and $\vec{r}_{\mathrm{cm}}(t)=\left(x_{\mathrm{cm}}(t)^{2}+y_{\mathrm{cm}}(t)^{2}+z_{\mathrm{cm}}(t)^{2}\right)^{1 / 2}$ denote the coordinate of the nanoparticle center of mass $(\mathrm{CM})$ at a time $t_{0}$ and at a later time $t$, respectively. The MSD 
is related to the diffusion coefficient $D$ by

$$
D=\lim _{t \rightarrow \infty} \frac{\left\langle\Delta \vec{r}_{\mathrm{cm}}(t)^{2}\right\rangle}{6 t} .
$$

The structure of the fluid was analyzed using the radial distribution function (RDF) $g\left(r_{i j}\right)$, and the pressure was evaluated with the virial expansion. In order to check if the Janus system shows density anomaly we evaluate the temperature of maximum density (TMD). Using thermodynamical relations, the TMD can be characterized by the minimum of the pressure versus temperature along isochores,

$$
\left(\frac{\partial p}{\partial T}\right)_{\rho}=0
$$

The fluid and micellar region in the $p \times T$ phase diagram were defined analyzing the structure with $g\left(r_{i j}\right)$, snapshots and the diffusion coefficient $D$.

\section{Results and Discussion}
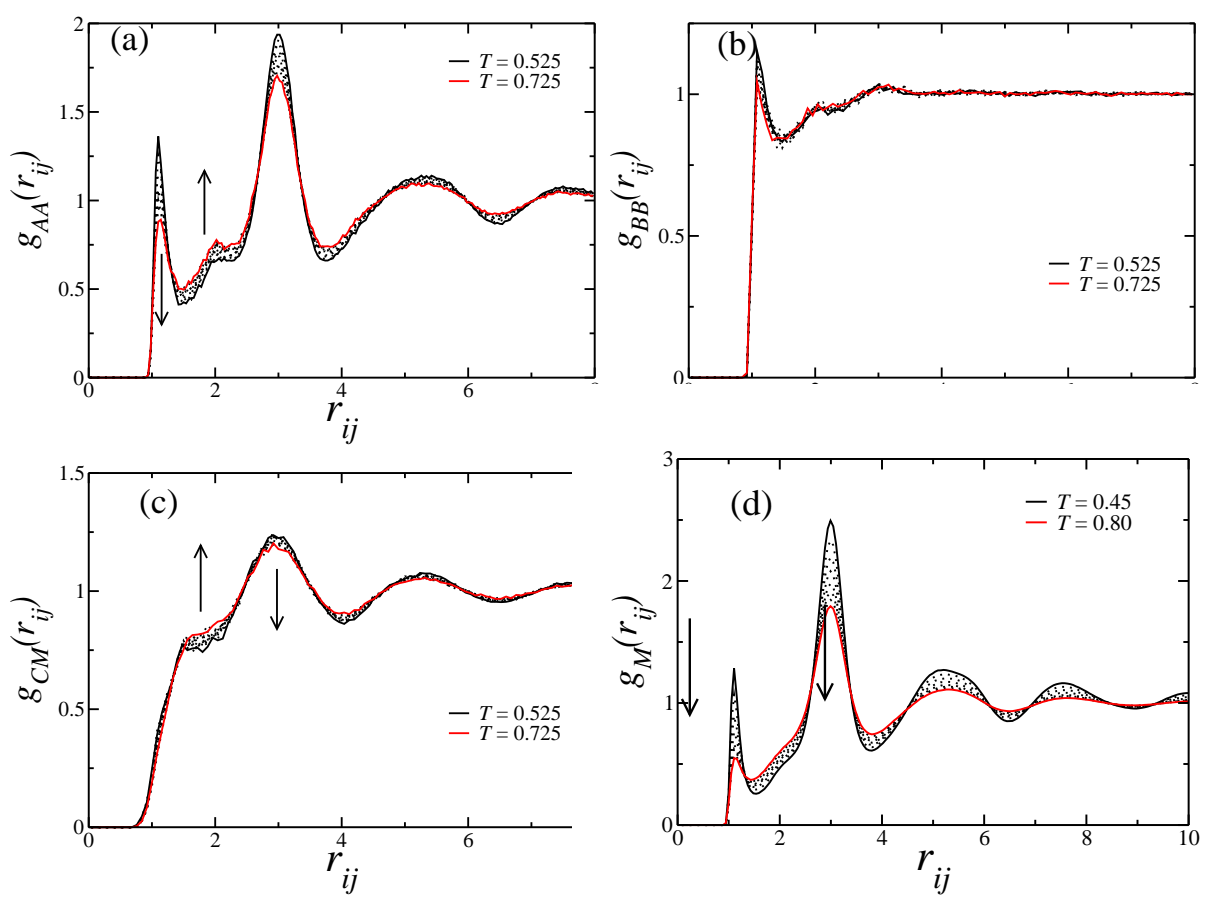

Figure 2: Radial distribution function between (a) A-A monomers, (b) B-B monomers and (c) nanoparticles center of mass at density $\rho=0.20$ and temperature ranging from $T=0.525$ to $T=0.725$. (d) RDF for the monomoric case at density $\rho=0.07$ and temperature ranging from $T=0.45$ to $T=0.80$.

Studies [19, 20] have shown that the main ingredient for a fluid present waterlike anomalies is not only 
the two length scales in the interaction, but due the competition between this scales. The TLS potential, equation 3, do not have density and diffusion anomalies in the monomeric case [17]. In this way, we first analyze the radial distribution function $g\left(r_{i j}\right)$ between A-A monomers, namely $g_{A A}\left(r_{i j}\right)$ and shown in figure 2 (a), B-B monomers, namely $g_{B B}\left(r_{i j}\right)$ and shown in figure 2 (b), and between the center of mass of each dimer, $g_{C M}\left(r_{i j}\right)$, figure $2(\mathrm{c})$.

The monomeric case, shown in figure 2 (d), shows that both peaks, related to the first and second shell, decreases when the temperature increases. This is the case where there is no competition between the scales, or there is no movement from one characteristic distance to another. Surprisingly, for the dumbbells nanoparticles we can observe a clear competition between the length scales.

The RDFs are shown for density $\rho=0.20$ and temperature between $T=0.525$ and $T=0.725$. The B particles RDF, $g_{B B}\left(r_{i j}\right)$, have a small competition between the scales as we increase the temperature at fixed density. On the other hand, $g_{A A}\left(r_{i j}\right)$ and $g_{C M}\left(r_{i j}\right)$ clearly have a movement from the particles from one scale to another. The $g_{A A}\left(r_{i j}\right)$ indicates that, as we increase the temperature, the particles leaves the first length scale, at $r_{i j} \approx 1.0$, and go to a second length scale at $r_{i j} \approx 2.0$. This is the distance where the fraction of imaginary modes from this family of TLS potentials has a local minimum [20]. The $g_{C M}\left(r_{i j}\right)$ shows the particles moving from a distance $r_{i j} \approx 3.0$ at lower temperatures to $r_{i j} \approx 2.0$ at the higher temperatures. This change in the RDF peaks, with one increasing while the other decreases, is a strong indicative that the system will have waterlike anomalies. Also, the RDF shows that not only the A monomers are arranging to go to the preferred distance, $r_{i j} \approx 2.0$, but the center of mass also moves to the distance $r_{i j} \approx 3.0$. This separation is equal to the second length scale plus the OLS monomer diameter, showing how the competition is generated by the dumbbell anisotropy.

Therefore, as a consequence from the competition between the two length scales the Janus dumbbell have density and diffusion anomalies. In figure 3 (a) we show the $p \times T$ phase diagram with the anomalies regions. The TMD is represented by the solid black line, the diffusion maxima and minima are represented by the red dashed line. The anomalous region does not have a waterlike hierarchy, and even an silicalike hierarchy. The diffusion anomalous region is at lower pressures (or lower densities) than the density anomalous region. Fomin and coworkers have shown that distinct hierarchy can be observed accordingly with the parameters used for core softened fluids [18, 46]. In this way, is possible to obtain the desired hierarchy with the potential parametrization.

Figure 3 (b) is the diffusion dependence with the system density, showing the anomalous increment in $D$ as we increase the fluid density. This results indicates that non-anomalous material can be combined to 

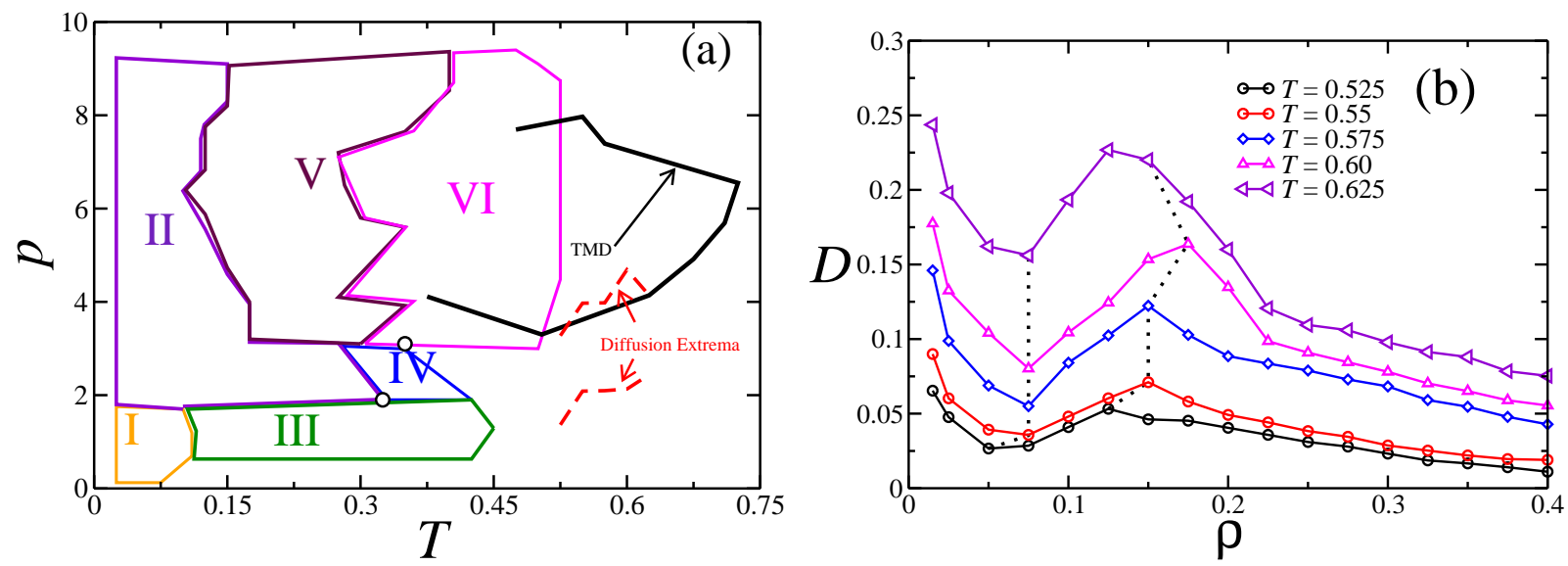

Figure 3: (a) Qualitative $p \times T$ phase diagram with the anomalies regions and the self-assembly structures and thermodynamic phases. Region I is a amorphous solid phase, with dimeric, trimeric and tetrameric micelles. Region II is a amorphous solid phase with spherical and wormlike micelles. Regions III correspond to a gas-liquid coexistence region, while region IV is a solid-fluid coexistence region. In the region V the system is structured in a lamellar solid micelles, and in region VI the system is structured in lamellar micelles with crystal-liquid characteristics. The two black circles corresponds to triple points. For simplicity, the isochores are not shown. (b) $D \times \rho$ curve, showing the diffusion maxima and minima.

create anomalous nanoparticles. The small attractive well in the LJ potential is located in the same place that the shoulder in the TLS potential, see figure 2 Essentially, the OLS monomers affects the competition between the length scales, taking the Janus dimer to have waterlike features.
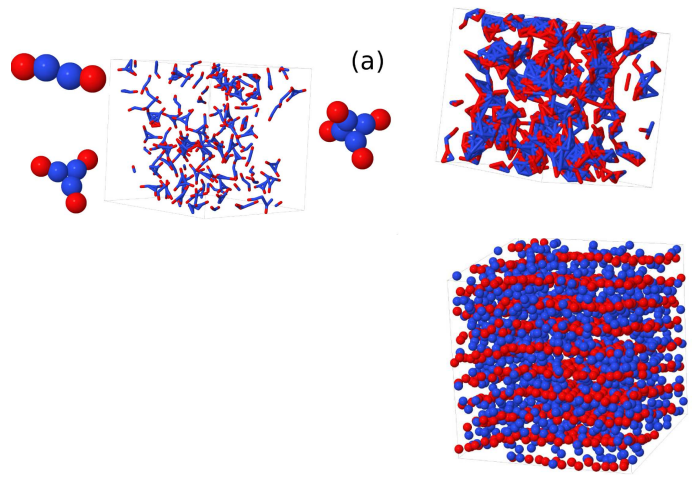

(b)

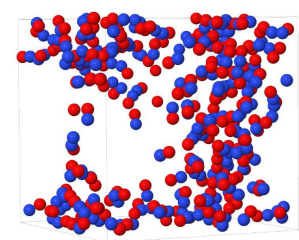

(e)

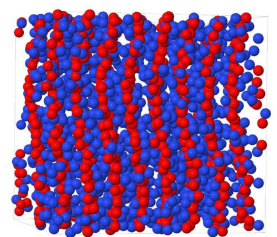

(c)

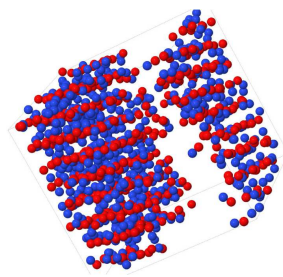

Figure 4: Snapshots of the observed structures and phases. (a) is in region I in the phase diagram, at $T=0.025$ and $\rho=0.075$, (b) is in the region II at $T=0.025$ and $\rho=0.25$, (c) is in region III at $T=0.10$ and $\rho=0.05$, (d) is in region IV at $T=0.40$ and $\rho=0.15$, (e) is in region $\mathrm{V}$ at $T=0.25$ and $\rho=0.30$ and (f) is in region $\mathrm{VI}$ at $T=0.50$ and $\rho=0.30$

Among the anomalous properties, the system exhibit several self-assembled structures. A region was defined as solid or fluid based in the nanoparticles structure, analyzed with the RDF and the snapshots, and based in the inclination of the MSD curve. With this, we should address that the phase diagram is 
qualitative, based on direct observation of the various assembled structures. At low temperatures and low densities, the orange region I in the $p \times T$ phase diagram, the nanoparticles are structured in an amorphous solid state, with the dimers assembled in dimeric, trimeric and tetrameric clusters. The structures observed are shown in figure 4 (a). Increasing the density, more nanoparticles will agregate in the same cluster. As consequence, spherical and elongated (wormlike) micelles were observed, as we shown in figure 4(b). Due the low temperature, the system remains in a amorphous solid state. In order to make the snapshot more clear, only the bonds inside each micelle are shown in this case. At intermediate temperatures and small densities, the system exhibits cavitation and a gas-liquid coexistence, region III in figure 4(a), as we shown in figure 4(c), while at the region IV there is a solid-fluid coexistence, figure 4(d). The holes in region III are similar to the observed in a model for alcohols [47] that uses a dimer related to our model. The solid in region IV corresponds to a lamellar phase, where the system is structured in planes, with a fluid hole. The lamellar structure was also observed in the regions V and VI, as discussed bellow in more details. The point where the regions II, III and IV connect, the lower circle in figure 3, can be understand as a triple point, since is the connection of 3 distinct regions. In fact, the point is crossed by several isochores, witch reinforces the existence of this triple point. For simplicity and in order to make the $p \times T$ phase diagram more clear we do not shown the isochores in figure 3

The region V delimit a lamellae region. The fluid is well structured in planes, as the figure 4 (e) shows. The same kind of structure was observed in the region VI, figure 4 (f). The A monomers are fixed related to each other, as the RDFs from figure 5 (a) and figure 5 (b) indicates, while the B monomers do not have a well defined structure. In a first moment, the RDFs and the snapshots could indicate that for the same isochore, $\rho=0.30$, in the region $\mathrm{V}(T=0.25)$ and VI $(T=0.50)$ the system have the same behavior. However, analyzing the nanoparticle center of mass MSD, shown in figure $5 \mathrm{c}$ ), we can see that at $T=0.25$ the system do not moves, as in a solid, while for $T=0.50$ the fluid have a large diffusion. In this way, in the region VI the fluid is structured in lamellar micelles, and moves in the direction of this planes. This planar liquid-crystal phase was already observed for dumbbells particles with potentials similar to the equation 3 , but without the attractive well [48]. Also, liquid-crystal was used in recent experiments for Janus dumbbells [41]. In the experimental system one monomer have a polymer compartment and a larger boundary. In fact, we can imagine this polymer compartment monomer as a TLS monomer, similar to our model. Also, a second triple point crossed by several isochores was found in the limits between the region IV, region VI and the region where the system is fluid.

The variety of self-assembled structures are not surprising. The micelles are present in hydrophobic- 

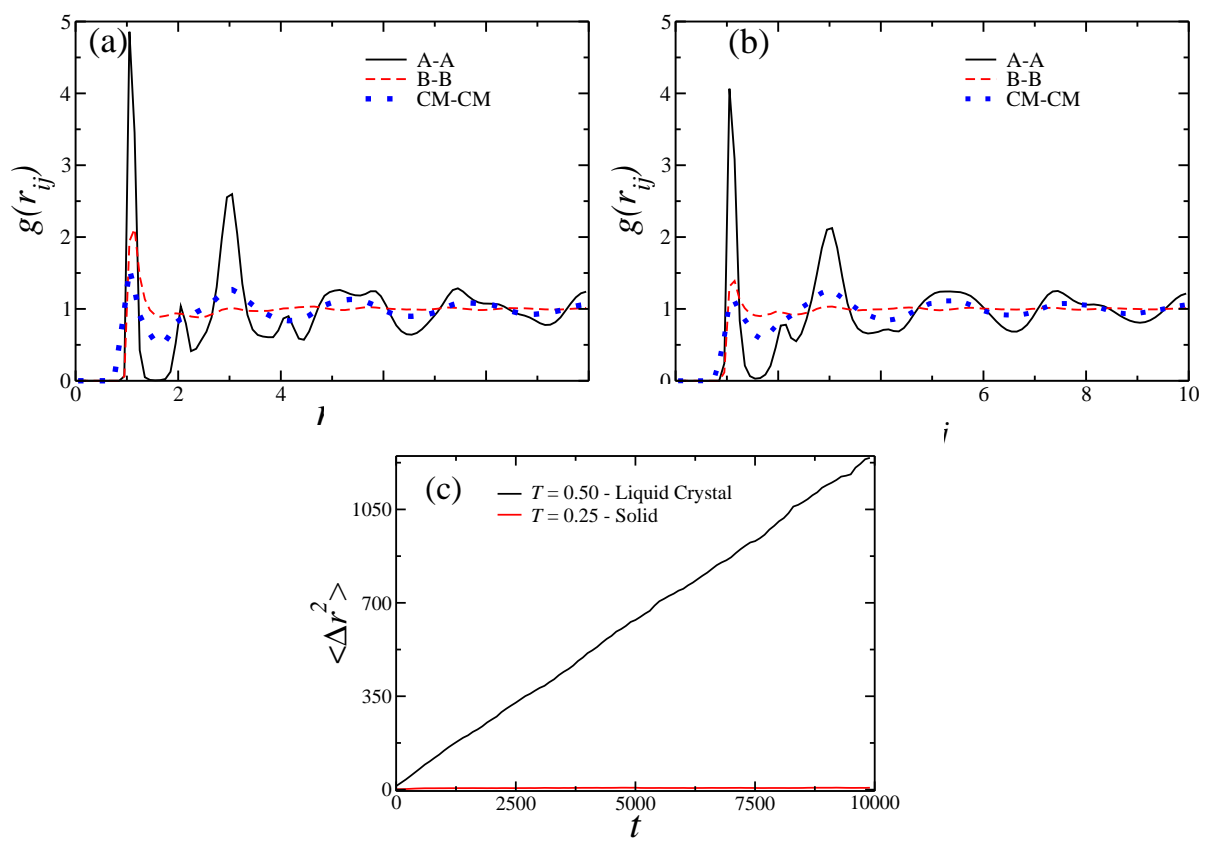

Figure 5: (a) RDF for the region (V) at $T=0.25$ and $\rho=0.30$ and (b) for the region (VI) at $T=0.50$ and $\rho=0.30$. The solid black line is the RDF between A monomers, the dashed blue line between B monomers and the dotted red line between the nanoparticles center of mass.(c) Nanoparticle center of mass MSD at $\rho=0.30$ for $T=0.25$, solid red line, and $T=0.50$, solid black line.

hydrophilic molecular systems, as surfactants and Janus particles. In our case, the competition between the TLS and OLS potential creates the micelles. As usual for competing interaction models, the transition between the fluid to structured phases are first-order.

\section{Conclusion}

The pressure versus temperature phase diagram of a Janus dumbbells model was studied. We analyze the effect of an attractive two length scales potential in the phase behavior. We have shown that, despite the monomeric system do not exhibit waterlike anomalies, the dimeric system with a OLS monomer and a TLS monomer will exhibit competition between the characteristic distances and, consequently, density and diffusion anomalies. The model shows a rich variety of micelles, similar to observed in hydrophobichydrophilic Janus dumbbells nanoparticles. Regions of gas-liquid and solid-fluid coexistence were found, both related with triple points. Also, a liquid-crystal phase was obtained, and the TMD line goes from the fluid region to the liquid-crystal region.

Our results indicates that is possible to create dimeric particles with anomalous properties using nonanomalous monomers, as colloids that have two characteristic scales for the interaction, and still have the 
self-assembly with different micellar conformations. Further investigation, including asymmetric monomers and distinct OLS/TLS potentials effects on anomalies and in the hierarchy are currently in progress.

\section{Acknowledgments}

We thank the Brazilian agency CNPq for the financial support.

\section{References}

\section{References}

[1] G. S. Kellu, Density, thermal expansivity, and compressibility of liquid water from 0.deg. to 150.deg.. correlations and tables for atmospheric pressure and saturation reviewed and expressed on 1968 temperature scale., J. Chem. Eng. Data 20 (1975) 97-105.

[2] C. A. Angell, E. D. Finch, P. Bach, Spin?echo diffusion coefficients of water to $2380 \mathrm{bar}$ and $-20^{\circ}$ c., J. Chem. Phys. 65 (1976) 3063

[3] M. Chaplin, Seventh-three anomalies of water, http://www.lsbu.ac.uk/water/anmlies.html (November 2015).

[4] S. Sastry, C. A. Angell, Liquid-liquidphase transition in supercooled silicon, Nature Mater. 2 (2003) 739-743.

[5] R. Sharma, S. N. Chakraborty, C. Chakravarty, Entropy, diffusivity, and structural order in liquids with waterlike anomalies, J. Chem. Phys. 125 (2006) 204501.

[6] H. Thurn, J. Ruska, Change of bonding system in liquid sexte1-1 alloys as shown by density measurements, J. Non-Cryst. Solids 22 (1976) 331.

[7] Handbook of Chemistry and Physics, 65th Edition, CRC Press, Boca Raton, Florida, 1984.

[8] S. J. Kennedy, J. C. Wheeler, On the density anomaly in sulfur at the polymerization transition, J. Chem. Phys. 78 (1983) $1523-1527$

[9] T. Tsuchiya, The anomalous negative thermal expansion and the compressibility maximum of molten ge-te alloys, J. Phys. Soc. Jpn. 60 (1991) 227-234.

[10] P. T. Cummings, G. Stell, Mean spherical approximation for a model liquid metal potential, Mol. Phys. 43 (1981) $1267-1291$.

[11] M. Togaya, Pressure dependences of the melting temperature of graphite and the electrical resistivity of liquid carbon, Phys. Rev. Lett. 79 (1997) 2474-2477.

[12] C. A. Angell, R. D. Bressel, M. Hemmatti, E. J. Sare, J. C. Tucker, Water and its anomalies in perspective: tetrahedral liquids with and without liquid-liquid phase transitions, Phys. Chem. Chem. Phys. 2 (2000) 1559.

[13] P. A. Netz, F. W. Starr, M. C. Barbosa, H. E. Stanley, Relation between structural and dynamical anomalies in supercooled water, Physica A 314 (2002) 470.

[14] T. Morishita, Anomalous diffusivity in supercooled liquid silicon under pressure, Phys. Rev. E 72 (2005) 021201.

[15] E. A. Jagla, Core-softened potentials and the anomalous properties of water, J. Chem. Phys. 111 (1999) 8980.

[16] A. B. de Oliveira, G. Franzese, P. Netz, M. C. Barbosa, Water-like hierarchy of anomalies in a continuous spherical shouldered potential, J. Chem. Phys. 128 (2008) 064901.

[17] J. N. da Silva, E. Salcedo, A. B. de Oliveira, M. C. Barbosa, Effects of the attractive interactions in the thermodynamic, dynamic and structural anomalies of a two length scale potential, J. Chem. Phys. 133 (2010) 244506.

[18] Y. D. Fomin, E. N. Tsiok, V. N. Ryzhov, Inversion of sequence of diffusion and density anomalies in core-softened systems, J. Chem. Phys. 135 (2011) 234502.

[19] N. M. Barraz Jr, E. Salcedo, M. C. Barbosa, Thermodynamic, dynamic and structural anomalies for shoulder-like potentials, J. Chem. Phys. 131 (2009) 094504.

[20] A. B. de Oliveira, E. Salcedo, C. Chakravarty, M. C. Barbosa, Entropy, diffusivity and the energy landscape of a water-like fluid, J. Chem. Phys. 132 (2010) 234509.

[21] M. Quesada-Pérez, A. Moncho-Jordá, F. Martínez-Lopez, R. Hidalgo-Álvarez, Probing interaction forces in colloidal monolayers: Inversion of structural data, J. Chem. Phys 115 (2001) 10897.

[22] C. Contreras-Aburto, J. M. Méndez-Alcaraz, R. C. neda Priego, Structure and effective interactions in parallel monolayers of charged spherical colloids, J. Chem. Phys 132 (2010) 174111.

[23] D. M. Talapin, J.-S. Lee, M. V. Kovalenko, E. V. Shevchenko, Prospects of colloidal nanocrystals for electronic and optoelectronic applications, Che. Rev. 110 (2010) 389-458.

[24] A. Elsukova, Z.-A. Li, C. Möller, M. Spasova, M. Acet, M. Farle, M. Kawasaki, P. Ercius, T. Duden, Structure, morphology, and aging of ag-fe dumbbell nanoparticles, Phys. Stat. Sol. 208 (2011) 2437-2442. 
[25] F. Tu, B. J. Park, D. Lee, Thermodynamically stable emulsions using janus dumbbells as colloid surfactants, Langmuir 29 (2013) 12679-12687.

[26] A. Walther, A. H. E. Müller, Janus particles, Soft Matter 4 (2008) 663-668.

[27] A. Walther, A. H. E. Müller, Janus particles: Synthesis, self-assembly, physical properties, and applications, Chem. Rev. 113 (2013) 5194-5261.

[28] J. Zhang, E. Luijten, S. Granick, Toward design rules of directional janus colloidal assembly, Annu. Rev. Phys. Chem. 66 (2015) 581-600.

[29] Y. Yin, Y. Lu, X. Xia, A self-assembly approach to the formation of asymmetric dimers from monodispersed spherical colloids, J. Am. Chem. Soc. 132 (2001) 771-772.

[30] V. Singh, C. Cassidy, P. Grammatikopoulos, F. Djurabekova, K. Nordlund, M. Sowwan, Heterogeneous gas-phase synthesis and molecular dynamics modeling of janus and core-satellite si-ag nanoparticles, J. Phys. Chem. C 118 (2014) 13869-13875.

[31] Y. Lu, Y. Yin, Z.-Y. Li, Y. Xia, Synthesis and self-assembly of au@ sio 2 core-shell colloids, Nano Lett. 2 (2002) 785-788.

[32] K. Yoon, D. Lee, J. W. Kim, D. A. Weitz, Asymmetric functionalization of colloidal dimer particles with gold nanoparticles, Chem. Cummun. 48 (2012) 9056-9058.

[33] Y. Liu, W. Li, T. Perez, J. D. Gunton, G. Brett, Self assembly of janus elipsoids, Langmuir 28 (2012) 3-9.

[34] B. Liu, C. Zhang, J. Liu, X. Qu, Z. Yang, Janus non-spherical colloids by asymmetric wet-etching, Chem Comm 26 (2009) 3871-3873.

[35] A. V. Nomoeva, A. R. Radnaeva, B. B. Baldanova, N. A. Torhovc, B. R. Radnaeva, N. A. Romanova, M. Schreibera, Thermodynamic considerations in the formation of janus-like tasi $2 /$ si nanoparticles by electron-beam evaporation, Chem. Phys. Lett. 637 (2015) 94-96.

[36] J. R. Bordin, L. B. Krott, M. C. Barbosa, Self-assembly and waterlike anomalies in janus nanoparticles, Langmuir 31 (2015) $8577-8582$.

[37] R. Deng, H. Li, F. Liang, J. Zhu, B. Li, X. Xie, Z. Yang, Soft colloidal molecules with tunable geometry by 3d confined assembly of block copolymers, Macromolecules 48 (2015) 5855-5866.

[38] K. H. Ku, Y. Kim, G.-R. Yi, Y. S. Jung, B. J. Kim, Soft patchy particles of block copolymers from interface-engineered emulsions, ACS Nano 9 (2015) 11333-11341.

[39] L. Jia, F. Zhou, W. Liu, Janus nanoparticle magic: selective asymmetric modification of au-ni nanoparticles for its controllable assembly onto attapulgite nanorods, Chem. Comm. 48 (2012) 12112-12114.

[40] J. Hu, S. Zhou, Y. Sun, X. Fang, L. Wu, Fabrication, properties and applications of janus nanoparticles, Chem. Soc. Rev. 41 (2012) 4356-4378.

[41] J. Jeong, A. Gross, W.-S. Wei, F. Tu, D. Lee, P. J. Collingsce, A. G. Yodhc, Liquid crystal janus emulsion droplets:preparation, tumbling, and swimming, Soft Matter 11 (2015) 6747-6754.

[42] P. Vilaseca, G. Franzese, Isotropic soft-core potentials with two characteristic length scales and anomalous behaviour, J. Non.Crys. Sol. 357 (2011) 519-426.

[43] P. Allen, D. J. Tildesley, Computer Simulation of Liquids, Oxford University Press, Oxford, 1987.

[44] H.-J. Limbach, A. Arnold, B. A. Mann, C. Holm, Espresso - an extensible simulation package for research on soft matter systems, Comput. Phys. Commun. 174 (2006) 704-727.

[45] A. Arnold, O. Lenz, S. Kesselheim, R. Weeber, F. Fahrenberger, D. Roehm, P. Koovan, C. Holm, Espresso 3.1: Molecular dynamics software for coarse-grained models, in: M. Griebel, M. A. Schweitzer (Eds.), Meshfree Methods for Partial Differential Equations VI, Vol. 89 of Lecture Notes in Computational Science and Engineering, Springer Berlin Heidelberg, 2013, pp. 1-23.

[46] Y. D. Fomin, E. N. Tsiok, V. N. Ryzhov, Silicalike sequence of anomalies in core-softened systems, Phys. Rev. E 87 (2013) 042122.

[47] G. Munaò, T. Urbic, Structure and thermodynamics of core-softened models for alcohols, J. Chem. Phys. 142 (2015) 214508.

[48] A. B. de Oliveira, E. Nevez, C. Gavazzoni, J. Z. Paukowski, P. A. Netz, M. C. Barbosa, Liquid crystal phase and water-like anomalies in a core-softened shoulder-dumbbells system, J. Chem. Phys. 132 (2010) 164505. 